\title{
VIBRATION CHARACTERISTICS ANALYSIS OF THE RUB- IMPACT ROTOR SYSTEM WITH MASS UNBALANCE
}

\author{
Jiu-Hong Jia and Tian-Qi Hang \\ Key Laboratory of Pressure Systems and Safety, Ministry of Education \\ East China University of Science and Technology, 200237 \\ Shanghai, PR China \\ jhjia@ecust.edu.cn
}

\begin{abstract}
In this paper, vibration characteristics of a rub-impact Jeffcott rotor system excited by mass unbalance including the eccentric mass and the initial permanent deflection are investigated. Through the numerical calculation, rotating speeds, mass eccentricities, initial permanent deflections and phase angles between the eccentric mass direction and the rotor initial permanent deflection direction are used as control parameters to investigate their effect on the rub-impact rotor system with the help of bifurcation diagrams, Poincare maps, frequency spectrums and orbit maps. Result shows that these two kinds of mass unbalance have great but different effect on the dynamic characteristic of the rubbing rotor system. Different motion characteristics appear with the varying of these control parameters, and complicated motions such as periodic and quasi-periodic vibrations are observed. Corresponding results can be used to diagnose the rub-impact fault and decrease the effect of rub impact in the rotor system with mass unbalance.
\end{abstract}

Key Words- Rub-impact rotor system, Mass unbalance, Nonlinear motion

\section{INTRODUCTION}

Mass unbalance of the rotor is the main factor to increase vibration and bring unstable characteristics to the whole rotor system [1-2]. Since the rotor-stator rub is one of the main faults for large rotary machines, it has attracted great concern and lots of research work has been done by many researchers. Cao and Ma [3] investigated nonlinear dynamic characteristics of rub-impact rotor system with fractional order damping. From the study, various complicated dynamic behaviors and types of routes to chaos were found, including period doubling bifurcation, sudden transition and quasiperiodic from periodic motion to chaos. Jawaidi [4] investigated dynamics of a rigid rotor supported by load-sharing between magnetic and auxiliary bearings for a range of realistic design and operating parameters. Many phenomena were found and studied. Zhang and Meng [5] carried out an analytical investigation on the stability of the rub 
solutions of the rotor system in MEMS. Numerical calculation demonstrated the complex nonlinear motion forms in this system.

Although much work has been done respectively on the research of rotor mass unbalance and rotor-stator rubbing impact, the vibration characteristics of this kind of rub-impact rotors with mass unbalance have rarely been studied specially in the literature. Due to mass unbalance's ubiquity, great effect on the vibration of the rubimpact rotor system and rub's large threat to the safe operation of the rotor system, Therefore, in this paper attention is paid to the research of vibration characteristics of rub-impact rotors with mass unbalance.

\section{PHYSICAL MODELAND EUATIONS}

Rub-impact forces in the $O$-xy coordinate system are [6]:

$$
\begin{aligned}
& F_{x}=-F_{N} \cos \gamma+F_{T} \sin \gamma=-(1-\mathrm{h} /|r|) K_{r}(x-\mu y) \\
& F_{y}=-F_{N} \sin \gamma-F_{T} \cos \gamma=-(1-\mathrm{h} /|r|) K_{r}(\mu x+y)
\end{aligned}
$$

when $|r|<h$, the rub doesn't happen. When $|r| \geq h$, the rub happens. Carrying out the nondimensional procedure, the governing motion equations are as follows:

When $\hat{R}=|r| / h<1.0$, the rub doesn't happen, and the nondimensional motion equations are:

$$
\begin{aligned}
& \hat{x}^{\prime \prime}+2 \xi \hat{x}^{\prime}+\hat{x}=\varepsilon \omega^{2} \cos \omega T+\hat{r}_{0} \cos \left(\omega T+\alpha_{0}\right) \\
& \hat{y}^{\prime \prime}+2 \xi \hat{y}^{\prime}+\hat{y}=\varepsilon \omega^{2} \sin \omega T+\hat{r}_{0} \sin \left(\omega T+\alpha_{0}\right)
\end{aligned}
$$

When $\hat{R}=|r| / h \geq 1.0$, the rub happens, and the nondimensional motion equations are:

$$
\begin{aligned}
& \hat{x}^{\prime \prime}+2 \xi \hat{x}^{\prime}+\hat{x}+\hat{K}(1-1 / \hat{R})(\hat{x}-\mu \hat{y})=\varepsilon \omega^{2} \cos \omega T+\hat{r}_{0} \cos \left(\omega T+\alpha_{0}\right) \\
& \hat{y}^{\prime \prime}+2 \xi \hat{y}^{\prime}+\hat{y}+\hat{K}(1-1 / \hat{R})(\mu \hat{x}+\hat{y})=\varepsilon \omega^{2} \sin \omega T+\hat{r}_{0} \sin \left(\omega T+\alpha_{0}\right)
\end{aligned}
$$

Where: $\hat{x}=x / h, \hat{y}=y / h, \hat{K}=K_{r} / K, \xi=C / 2 \omega_{0} M, \omega_{0}=\sqrt{K / M}$ the natural frequency of the shaft, $\omega=\Omega / \omega_{0}$ the frequency ratio, $\varepsilon=e_{m} / h, \hat{r}_{0}=r_{0} / h, T=\omega_{0} t,{ }^{\prime}=d / d T$.

Since the nondimensional governing equations of motion have been got above, they are transferred into a set of first order differential equations $\dot{u}=f(u)$. Then the fourth-order Runge-Kutta method is used to integrate this set of equations. According to the analysis need, some parameters can be used as the control parameters such as the rotor rotating speed, the initial permanent deflection and so on, while other parameters keep fixed during every time of calculation. To get the stable result, a small integration 
step has to be chosen to avoid the numerical divergence at the point where derivatives of $F_{x}$ and $F_{y}$ are discontinuous. In this paper, the integration step is chosen to be $2 \pi / 500$, i.e., within one period, there are 500 times of integral calculation. Generally, long time marching computation is required to obtain a convergent orbit. In this paper, during every calculation, results of the first 500 periods are abandoned, and then results of the next 100 periods are got to carry out various kinds of analysis. To study the vibration characteristics of the rubbing rotor system with mass unbalance, bifurcation diagrams, Poincare maps, frequency spectrums and orbit maps are employed. They are all useful and effective ways to illustrate the motion behavior of the rotor system.

\section{NUMERICAL SIMULATION OF MOTIONS OF THE ROTOR SYSTEM}

\subsection{Effect of Rotor Rotating Speeds}

Although many parameters such as the system damping and the frictional coefficient between the rotor and the stator can be used to investigate the vibration characteristics of the rub-impact system, the most common and useful parameter used is the rotor rotating speed. The whole starting process can be observed by using the rotating speed to simulate the rub-impact rotor system. The parameters used during computations are: $\xi=0.12, \hat{K}=30.0, \mu=0.12$.

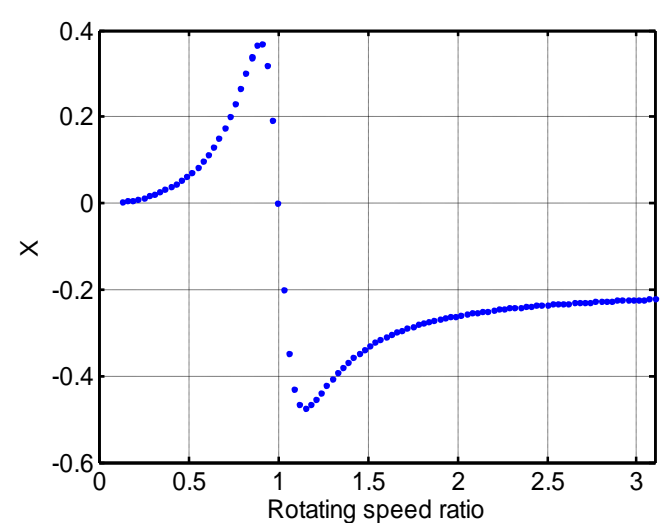

(a) $\varepsilon=0.2$

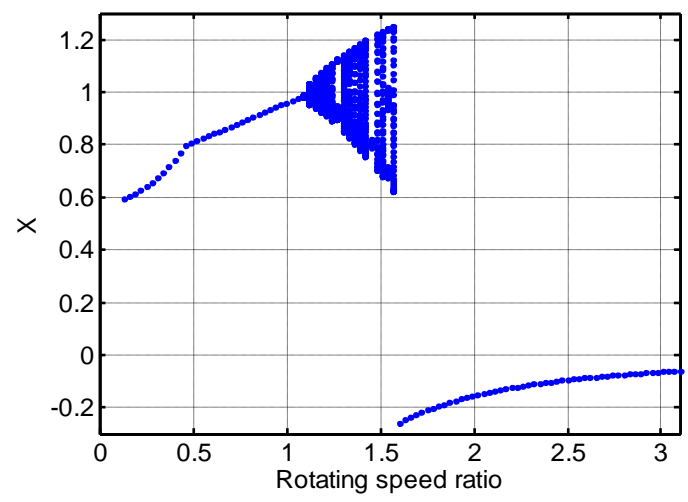

(b) $\hat{r}_{0}=0.8, \alpha_{0}=\pi / 4$; 


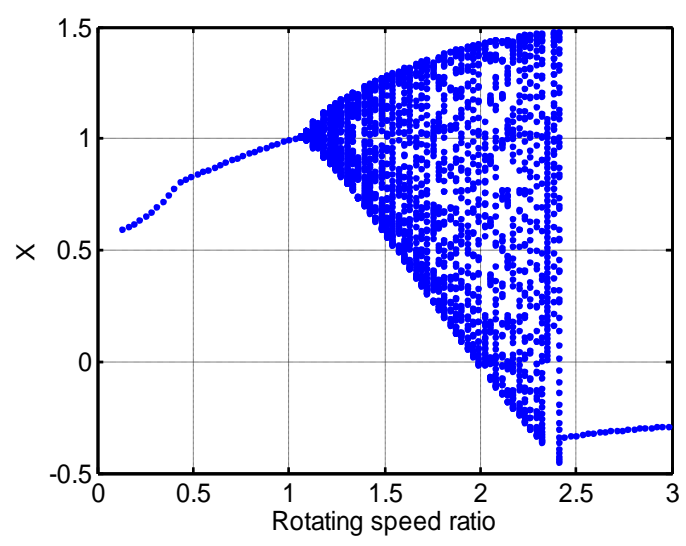

(c) $\hat{r}_{0}=0.8, \alpha_{0}=\pi / 4, \varepsilon=0.2$

Figure 1. Bifurcation diagram

Fig.1.(a) is the bifurcation diagram of the rub-impact rotor's vibration which is excited only by the eccentric mass. Fig.1.(b) is the bifurcation diagram of the rubimpact rotor's vibration which is excited only by the initial permanent deflection. Fig.1.(c) is the bifurcation diagram of the vibration excited by these two kinds of mass unbalance jointly. Very clearly from Fig.1, different shapes of three diagrams demonstrate that the eccentric mass and the initial permanent deflection have different influence to the vibration of the rubbing rotor system and both contribute a lot to the vibration characteristics of the rub-impact rotor system. As shown in Fig.1.(a), the rubimpact motion excited only by the eccentric mass keeps synchronous with period-one for the whole calculating rotating speed range. Fig.2 shows that only one point is correspondingly shown in the Poincare map, only one peak appears in the frequency spectrum, and the orbit of the rotor center is an ellipse. All these further demonstrate the motion is period-one motion. From Fig.1.(b), it can be seen that when the rotating speed ratio is from 0.0 to 1.1 , the motion is period-one. From 1.1 to 1.6 , the motion is quasiperiodic, then the motion transits to period-one motion again for the higher speed ratios. This can be seen from Fig.3. It illustrates the rubbing motion excited only by the initial permanent deflection at $\omega=1.2$. The Poincare map is a closed circle, several peaks appear in the frequency spectrum, and the rotor center orbit is irregular. These prove that the motion is quasi-periodic.

From Fig.1.(c), it can be seen that when the rotating speed ratio is from 0.0 to 1.1, the motion is period-one, from 1.1 to 2.4 , the motion is quasi-periodic, then the motion transits to period-one motion again for the higher speed ratios. This motion type is clearly different from the one excited only by the eccentric mass in Fig.1.(a) and the one excited only by the initial permanent deflection in Fig.1.(b). Fig.4 demonstrates that the motion in this case at $\omega=1.5$ is quasi-periodic as analyzed above. 


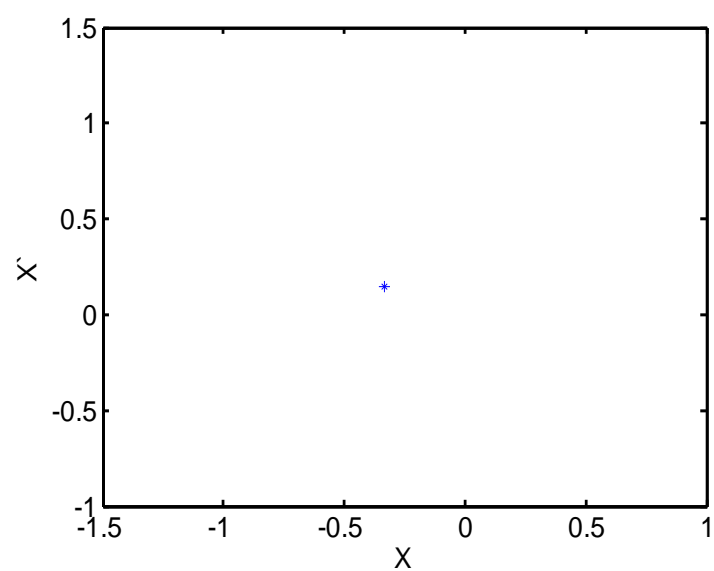

(a) Poincare map

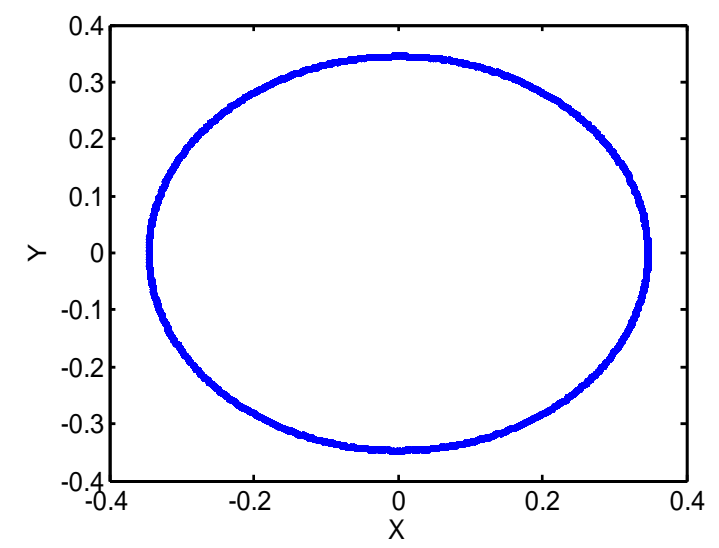

(b) orbit

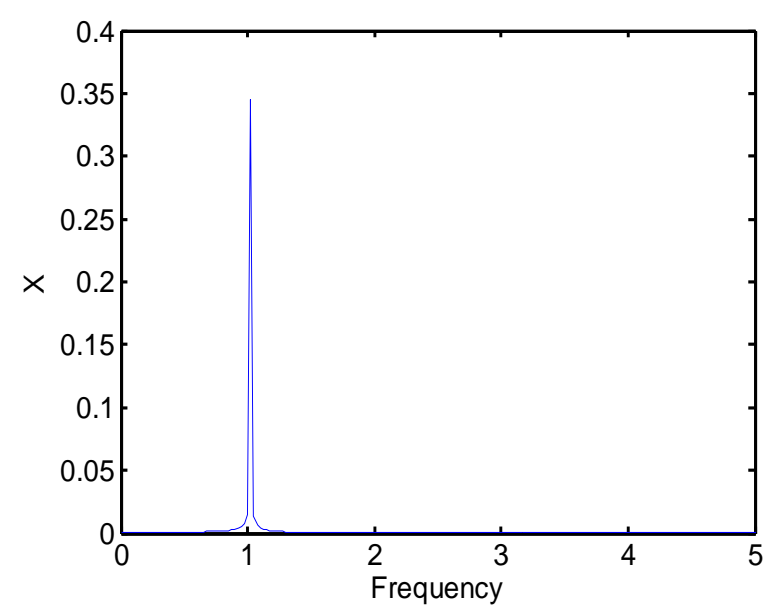

(c) spectrum of frequency

Figure 2. Motion excited by $\varepsilon=0.2$ at $\omega=1.5$

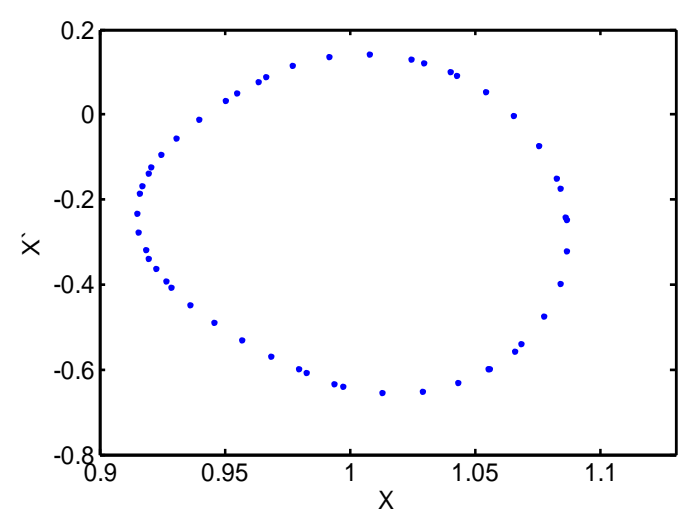

(a) Poincare map

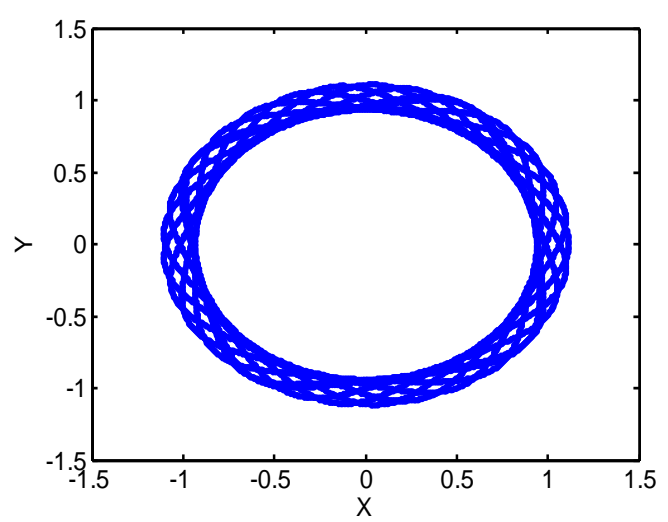

(b) orbit 


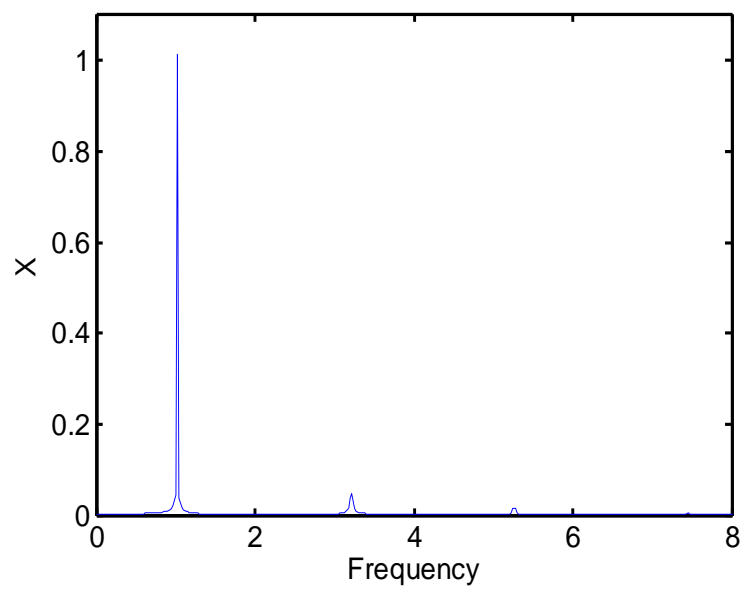

(c) spectrum of frequency

Figure 3. Motion excited by $\hat{r}_{0}=0.8, \alpha_{0}=\pi / 4$ at $\omega=1.2$

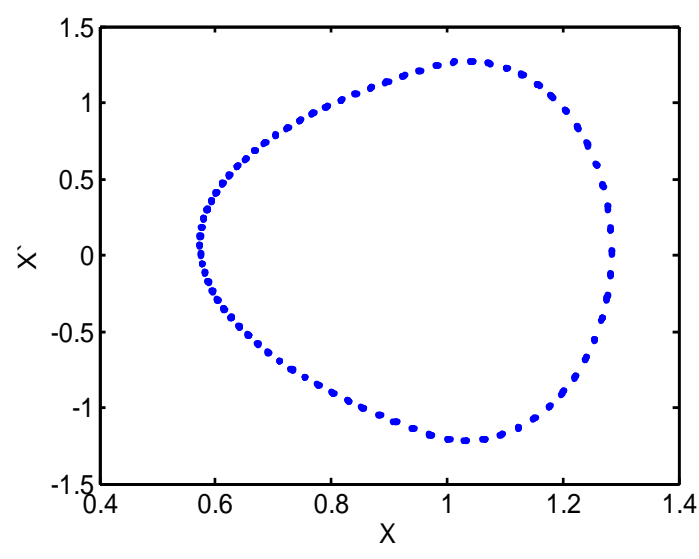

(a) Poincare map

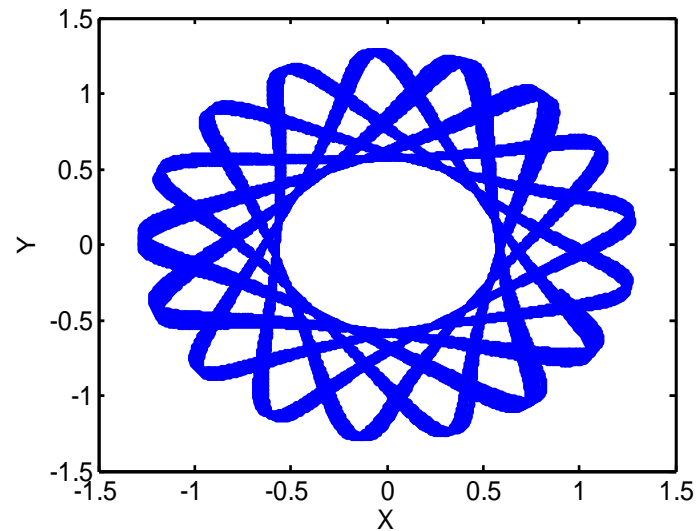

(b) orbit

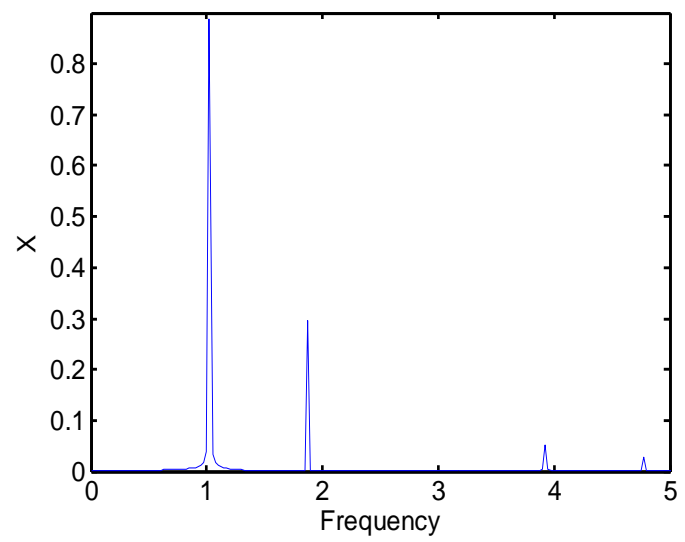

(c) spectrum of frequency

Figure 4. Motion excited jointly by $\varepsilon$ and $\hat{r}_{0}$ at $\omega=1.5$ 


\subsection{Effect of the Eccentric Mass}

Eccentric mass is one of the mass unbalance to excite the motion of the rotor system, so it's essential to study its effect on dynamic characteristic of the rub-impact rotor system. Fig. 3 are bifurcation diagrams of rotor's vibration at different rotating speeds using the mass eccentricity ratio $\varepsilon$ as the control parameter. The initial permanent deflection is not included in this analysis and is set as 0.0 .

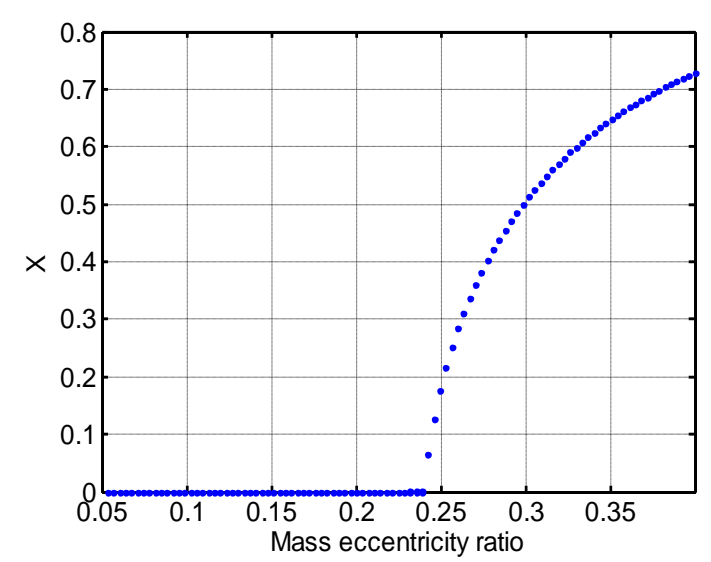

(a) $\omega=1.0$

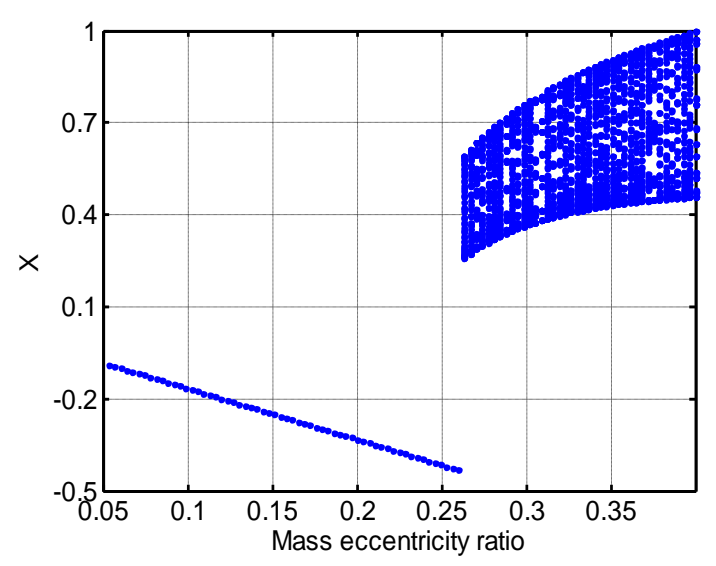

(b) $\omega=1.5$

Figure 5. Bifurcation diagram using $\varepsilon$ as the control parameter

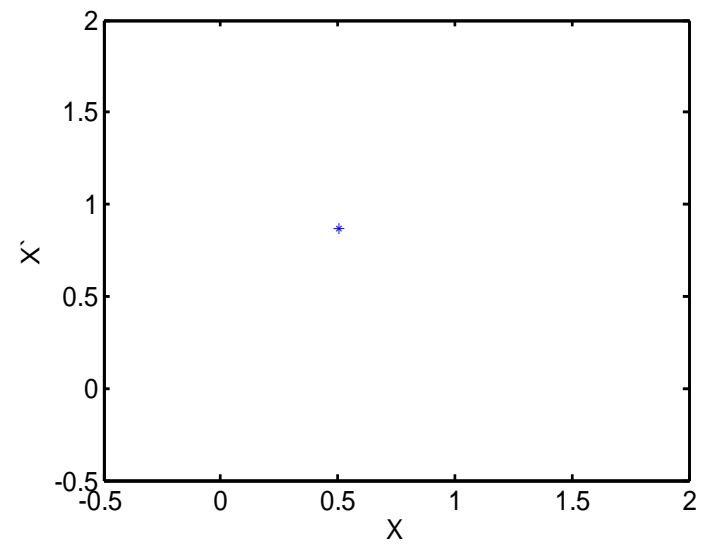

(a) Poincare map

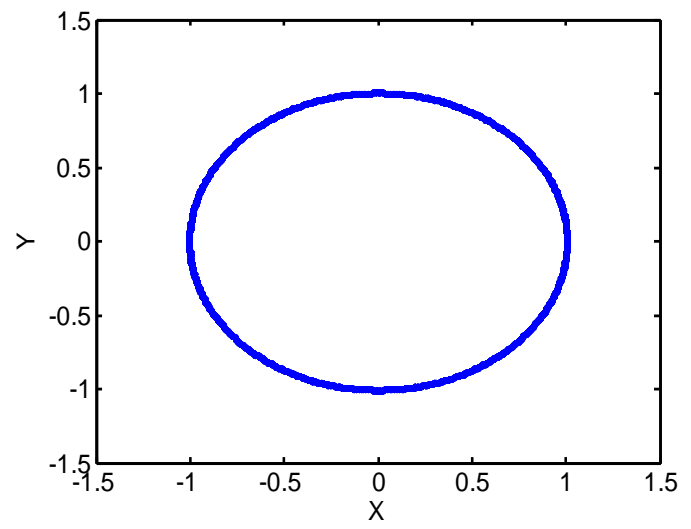

(b) orbit 


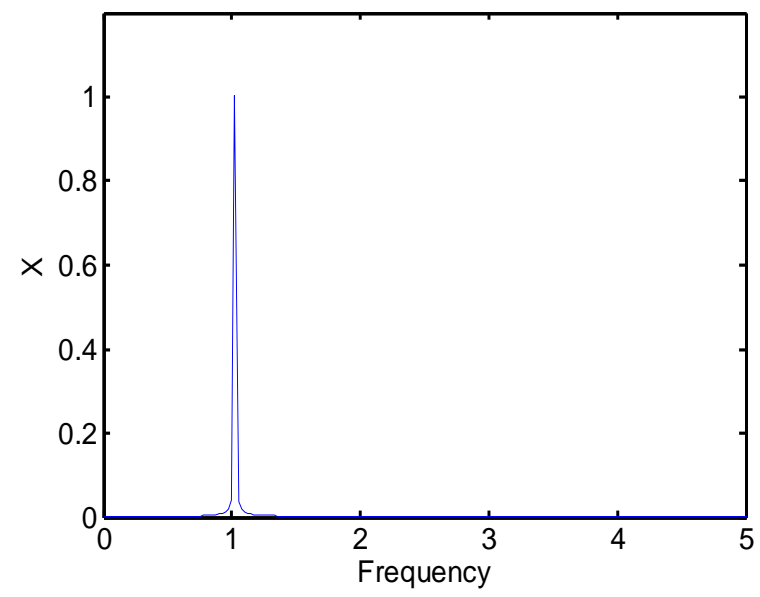

(c) spectrum of frequency

Figure 6. Motion with $\varepsilon=0.3$ at $\omega=1.0$

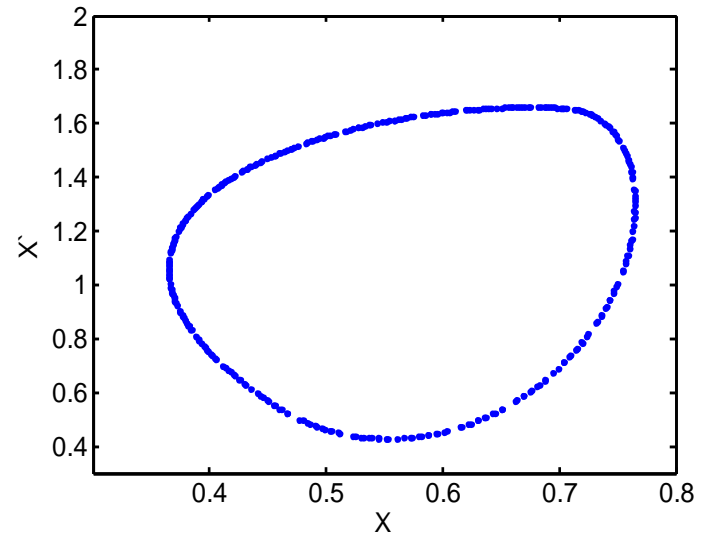

(a) Poincare map

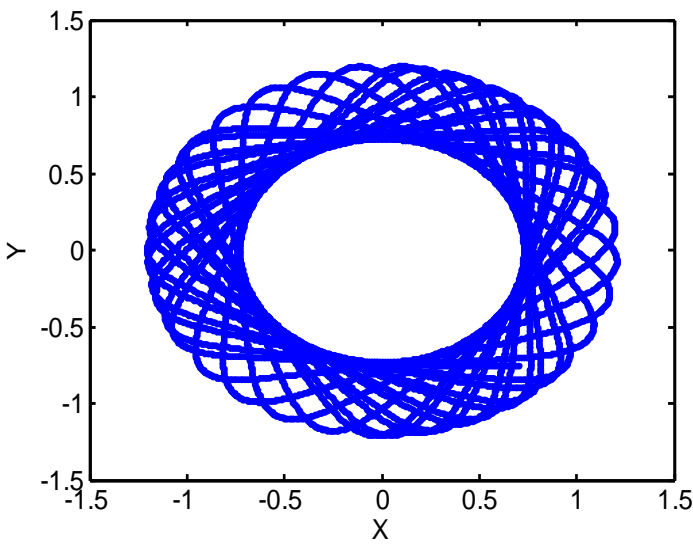

(b) orbit 


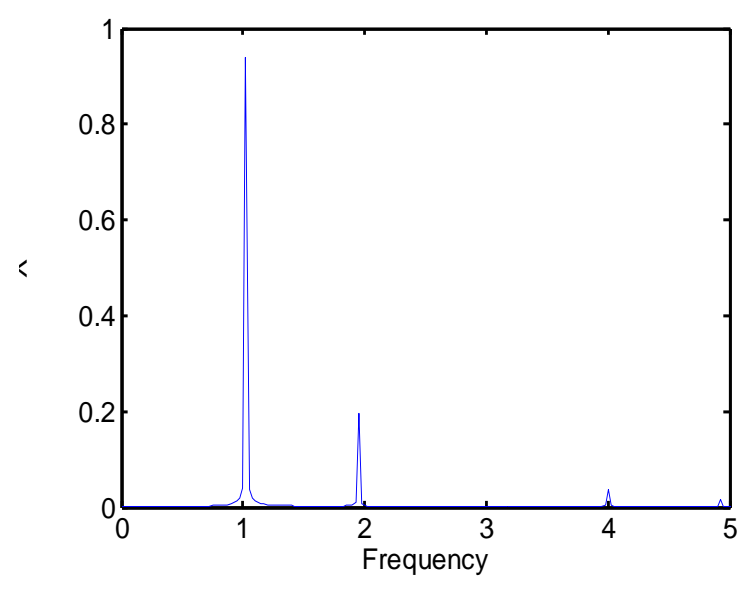

(c) spectrum of frequency

Figure 7. Motion with $\varepsilon=0.3$ at $\omega=1.5$

As shown in Fig.5.(a), when the rotating speed ratio is not high like 1.0, the motion of the rub-impact rotor system keeps synchronous with period-one for the whole calculating range. Further demonstration is shown in Fig.6 with $\varepsilon=0.3$ at $\omega=1.0$ that only one point is correspondingly shown in the Poincare map, only one peak appears in the frequency spectrum, and the orbit of the rotor center is an ellipse. All these further demonstrate the motion is period-one motion.

As shown in Fig.5. (b), at $\omega=1.5$ when the mass eccentricity ratio $\varepsilon$ is from 0.0 to 0.26 , the motion is period-one. From 0.26 to higher values of the mass eccentricity ratio, the motion keeps quasi-periodic. In Fig.7 with $\varepsilon=0.3$ at $\omega=1.5$, the Poincare map is a closed circle, several peaks appear in the frequency spectrum, and the rotor center orbit is irregular. These prove that the motion is quasi-periodic.

\subsection{Effect of the Initial Permanent Deflection}

The initial permanent deflection is another one of the mass unbalance to excite the motion of the rotor system, it's also important to study its effect on dynamic characteristic of the rub-impact rotor system. Fig.8 is bifurcation diagrams of rotor's vibration at different rotating speeds using the initial permanent deflection ratio $\hat{r}_{0}$ as the control parameter. The mass eccentricity is not included in this analysis and is set as 0.0. $\alpha_{0}$ is fixed as $\pi / 4$. 


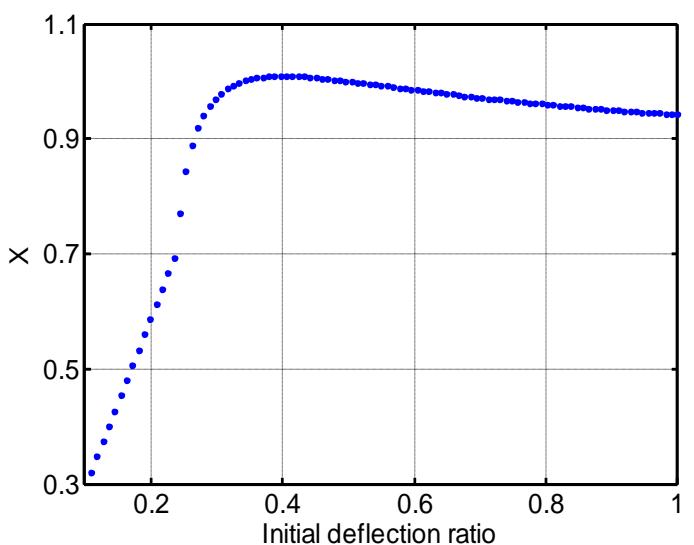

(a) $\omega=1.0$

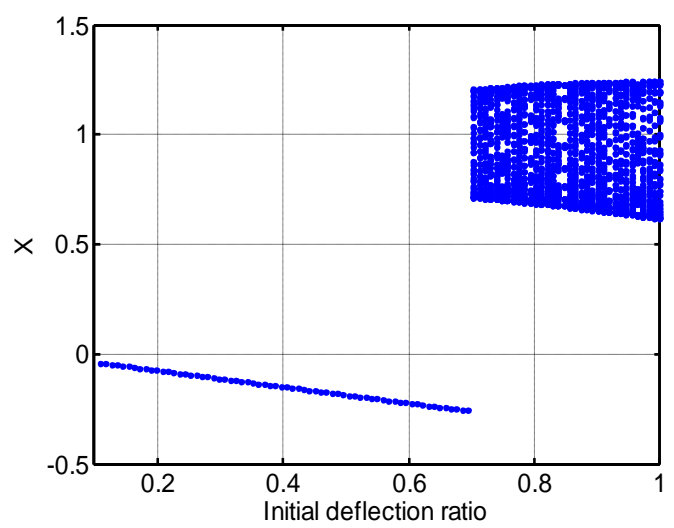

(b) $\omega=1.5$

Figure 8 . Bifurcation diagram using $\hat{r}_{0}$ as the control parameter

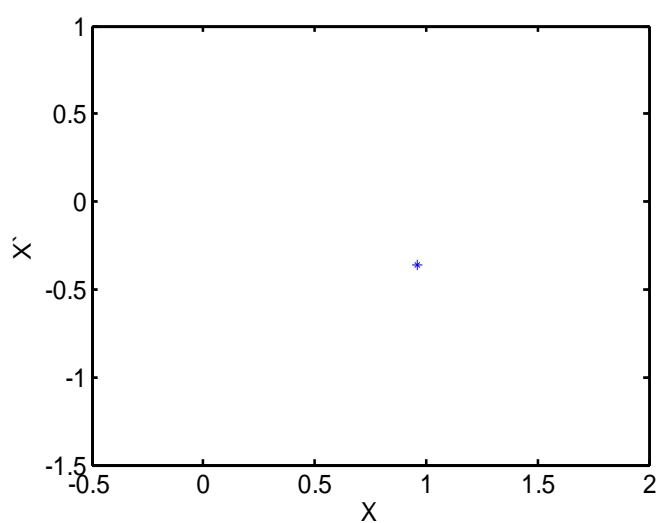

(a) Poincare map

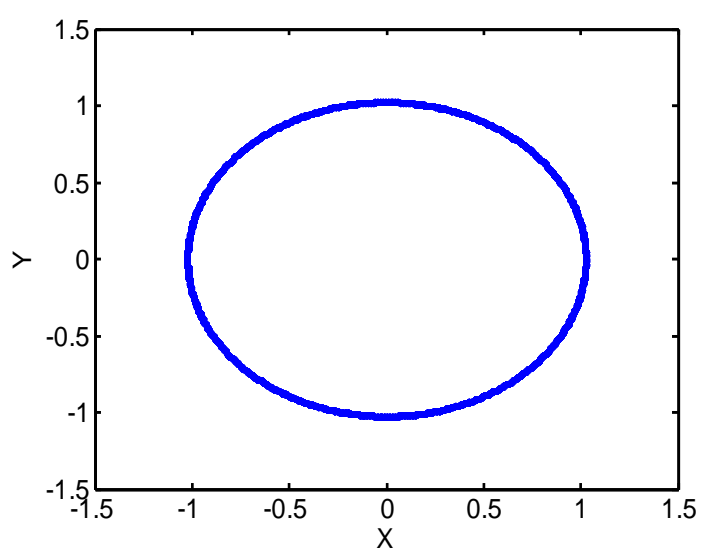

(b) orbit

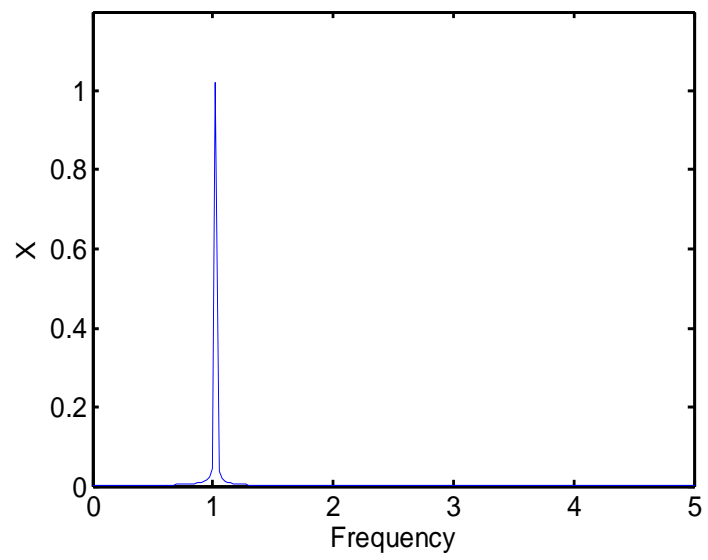

(c) spectrum of frequency

Figure 9. Motion with $\hat{r}_{0}=0.8$ at $\omega=1.0$ 


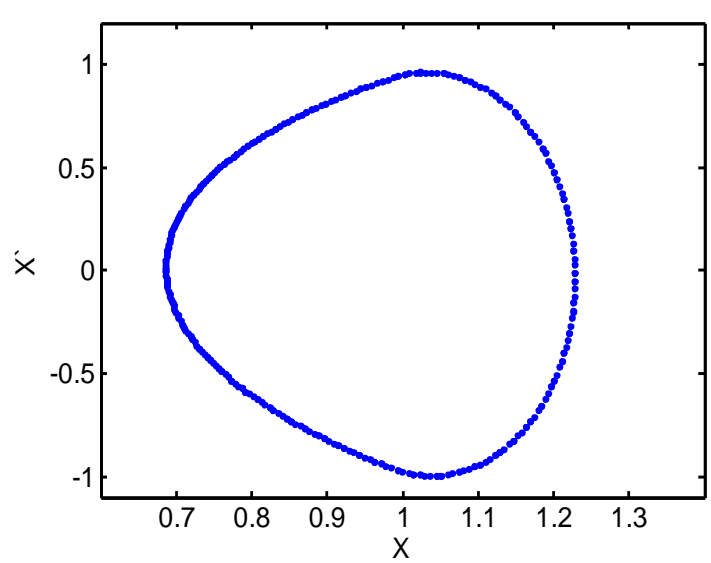

(a) Poincare map

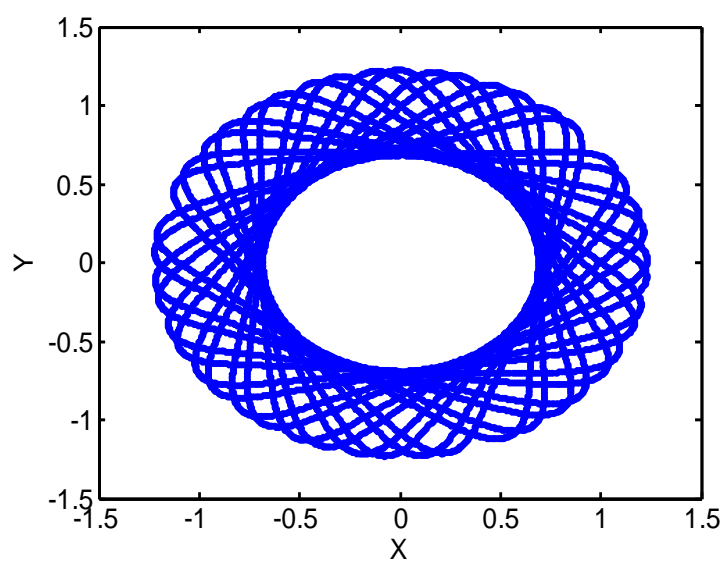

(b) orbit

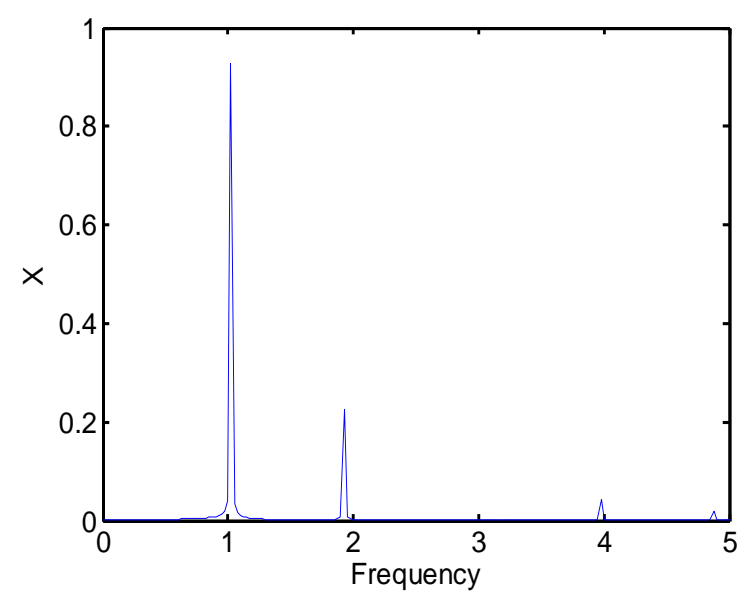

(c) spectrum of frequency

Figure 10. Motion with $\hat{r}_{0}=0.8$ at $\omega=1.5$

As shown in Fig.8.(a), when the rotating speed ratio is at 1.0, the motion of the rub-impact rotor system keeps synchronous with period-one for the initial deflection ratio ranging from 0.0 to 1.0. Further demonstration is shown in Fig. 9 with $\hat{r}_{0}=0.8$ at $\omega=1.0$.

As shown in Fig.7.(b), at $\omega=1.5$ when the initial permanent deflection ratio $\hat{r}_{0}$ is from 0.0 to 0.7 , the motion is period-one. From 0.7 to higher values of the initial permanent deflection ratio, the motion keeps quasi-periodic. Further demonstration is shown in Fig. 10 with $\hat{r}_{0}=0.8$ at $\omega=1.5$.

\subsection{Effect of Phase Angles between the Eccentric Mass Direction and the Rotor Deflection Direction}

As shown in above equations (2) and (3), the phase angle between the eccentric mass direction and the rotor deflection direction is an important factor to influence the 
amplitude of the rotor center, so it's meaningful to study its effect on the rub-impact vibration characteristics of the rotor system. Fig.11 are the bifurcation diagrams of the rotor vibration at different rotating speed ratio $\omega$ using the phase angle $\alpha_{0}$ as the control parameter with $\hat{r}_{0}=0.8, \varepsilon=0.2$.

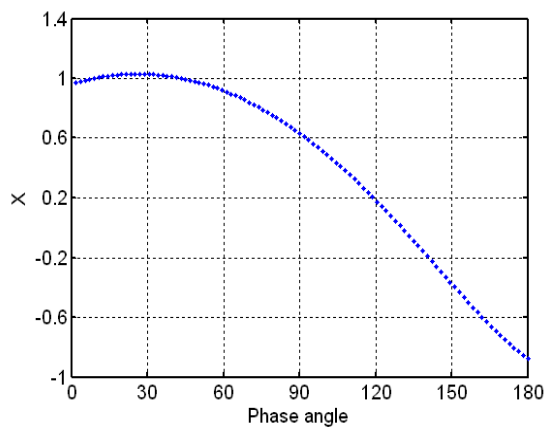

(a) $\omega=1.0$

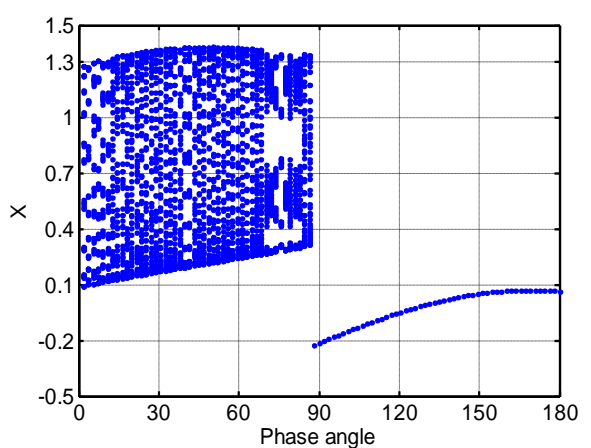

(c) $\omega=1.8$

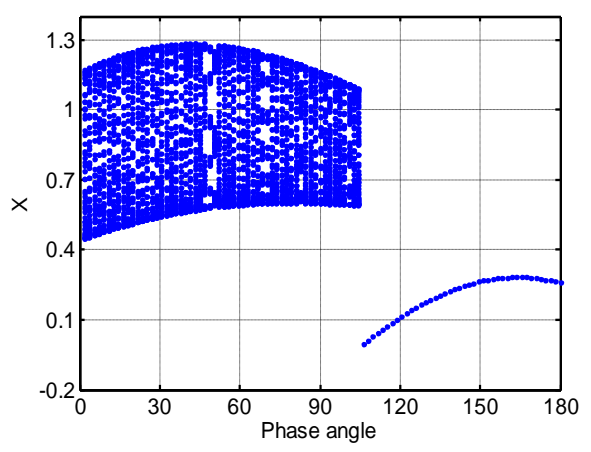

(b) $\omega=1.5$

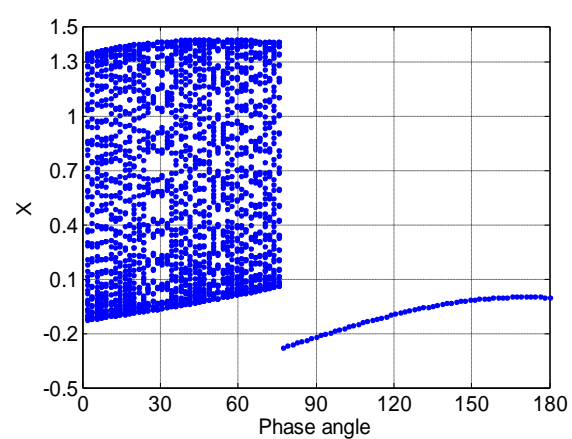

(d) $\omega=2.0$

Figure 11. Bifurcation diagram using $\alpha_{0}\left({ }^{\circ}\right)$ as the control parameter

From Fig.11.(a), when $\omega=1.0$, the motion of the rub-impact rotor system keeps synchronous with period-one for the calculating phase angle ranging from 0.0 to $180^{\circ}$. From Fig.11.(b), when $\omega=1.5$, for the phase angle from 0.0 to $105^{\circ}$, the motion is quasiperiodic. Form $95^{\circ}$ to $180^{\circ}$, the motion transits to period-one motion. Shown in Fig.11.(c), when $\omega$ increases up to 1.8. the motion keeps quasi-periodic for the phase angle ranging from 0.0 to $86^{\circ}$, then transits to period-one for the rest phase angle. In Fig. 11. (d), when $\omega=2.0$, the quasi-periodic motion range is for the phase angle from 0.0 to $75^{\circ}$, the motion for the rest phase angle is period-one. A clear trend can be seen from above analysis that with the increase of rotating speeds, the quasi-periodic motion range declines, correspondingly the period-one motion range increases. 


\section{CONCLUSIONS}

In this paper, vibration characteristics of a rub-impact Jeffcott rotor system excited by mass unbalance including the eccentric mass and the initial permanent deflection are investigated. From numerical calculation, rotating speeds, mass eccentricities, initial permanent deflections and phase angles between the eccentric mass direction and rotor initial permanent deflection direction are used as the control parameters to investigate their effect on the rub-impact rotor system. Result shows that these two kinds of mass unbalance: eccentric mass and initial permanent deflection have great but different effect on the dynamic characteristic of the rubbing rotor system. Periodic, quasiperiodic motions appear with the varying of these control parameters. Because the quasi-periodic motion is the route to chaos, it indicates that the system has the potential to become chaotic. These results can be used to diagnose the rub fault and direct the work to decrease the effect of rub impact in the rotor system with mass unbalance.

Acknowledgements- The authors are grateful for the support of National natural science foundation of China (NO.50905059) and new teacher fund of doctorial program sponsored by Ministry of Education of China (20090074120005).

\section{REFERENCES}

1. Y. H. Liao, and P. Zhang, Unbalance related rotor precession behavior analysis and modification to the holobalancing method. Mechanism and Machine Theory 45, 601610. 2010.

2. A. K. Jalan and A.R. Mohanty, Model based fault diagnosis of a rotor-bearing system for misalignment and unbalance under steady-state condition. Journal of Sound and Vibration 327, 604-622, 2009.

3. J. Y. Cao, C. B. Ma, etc. Nonlinear dynamic analysis of fractional order rub-impact rotor system. Communications in Nonlinear Science and Numerical Simulation 16, 1443-1463, 2011.

4. I. H. Jawaidi, Nonlinear dynamics of a magnetically supported rigid rotor in auxiliary bearings. Mechanism and Machine Theory 45, 1651-1667, 2010.

5. W. M. Zhang and G. Meng. Stability, bifurcation and chaos of a high-speed rubimpact rotor system in MEMS. Sensors and Actuators A 127, 163-178, 2006.

6. X. Y. Shen, J. H. Jia and M. Zhao, Nonlinear analysis of a rub-impact rotor-bearing system with initial permanent rotor bow, Archive of Applied Mechanics 78, 225-240, 2008. 